\title{
On the graph of a function in two variables over a finite field
}

\author{
Simeon Ball • Michel Lavrauw
}

Received: October 21, 2004 / Revised: September 15, 2005 / Accepted: September 19, 2005

(C) Springer Science + Business Media, Inc. 2006

\begin{abstract}
We show that if the number of directions not determined by a pointset $\mathcal{W}$ of $\operatorname{AG}(3, q), q=p^{h}$, of size $q^{2}$ is at least $p^{e} q$ then every plane intersects $\mathcal{W}$ in 0 modulo $p^{e+1}$ points and apply the result to ovoids of the generalised quadrangles $T_{2}(\mathcal{O})$ and $T_{2}^{*}(\mathcal{H})$.
\end{abstract}

Keywords Directions determined by a function - Directions determined by a set . Generalised quadrangles · Ovoids

\section{Preliminaries}

Let $\mathrm{AG}(n, q)$, respectively $\mathrm{PG}(n, q)$, denote the affine, respectively projective, $n$-dimensional space over the finite field $\operatorname{GF}(q)$ with $q$ elements, where $q=p^{h}$ for some prime $p$. Let $f$ be a function from $\operatorname{GF}(q)^{2}$ to $\operatorname{GF}(q)$ and let

$$
\mathcal{W}_{f}:=\{\langle a, b, f(a, b), 1\rangle: a, b \in \mathrm{GF}(q)\},
$$

be the set of points corresponding to the graph of the function $f$ in $\operatorname{AG}(3, q)$. Let $\pi$ be the plane with equation $X_{3}=0$, and put

$$
\mathcal{D}(f):=\left\{\langle P, Q\rangle \cap \pi: P, Q \in \mathcal{W}_{f}, P \neq Q\right\} .
$$

We call $\mathcal{D}(f)$ the set of directions determined by $f$. Often we will only refer to the set of affine points $\mathcal{W}_{f}$ and talk about the number of directions determined by $\mathcal{W}_{f}$ instead of by $f$. Note that $\left|\mathcal{W}_{f}\right|=q^{2}$ and that for any set $\mathcal{W}$ of $q^{2}$ affine points in $\operatorname{PG}(3, q)$ one can define a function $f_{\mathcal{W}}$ provided that $\mathcal{W}$ does not determine every direction. The main result of this paper is that if the number of directions not determined by $\mathcal{W}$ is more than $q$ then every plane of $\operatorname{PG}(3, q)$ intersects $\mathcal{W}$ in 0 modulo $p$ points. After the proof of this result, we will prove

S. Ball $(\bowtie)$ M. Lavrauw

Departament de Matemàtica Aplicada IV, Universitat Politècnica de Catalunya, Jordi Girona 1-3, Mòdul C3, Campus Nord, 08034 Barcelona, Spain e-mail: simeon@mat.upc.es, lavrauw@mat.upc.es 
two more theorems, by refining the hypotheses in one case and for $p=2$ in the other case. In the last section we consider some consequences for ovoids of the generalised quadrangles $T_{2}(\mathcal{O})$ and $T_{2}^{*}(\mathcal{H})$, where $\mathcal{O}$ is an oval and $\mathcal{H}$ is a hyperoval of $\operatorname{PG}(2, q)$. In the special case where $\mathcal{O}$ is a conic, these consequences are similar to those obtained in [4].

In contrast to the main result of this article, Storme and Sziklai [8] prove that if the number of directions determined by $\mathcal{W}$ is less than $q(q+3) / 2$ then every line is incident with exactly one point of $\mathcal{W}$ or $0 \bmod p$ points. If $p>3$ they prove that $\mathcal{W}$ is $\operatorname{GF}(s)$-linear for some subfield $\mathrm{GF}(s)$ of $\mathrm{GF}(q)$. Their proof uses the main result in [5] which classifies those sets of $q$ points in $\operatorname{AG}(2, q)$ that determine less than half the directions. This problem dates back to Rédei [7, pp. 226], who together with Megyesi solved the prime case, and has now been solved completely, for the most part in [5] and for characteristic two and three in [2]. The restriction $p>3$ in [8] can been weakened to $p>2$ as a result of [2].

\section{The number of directions}

We start with a lemma concerning the number of zeros of a polynomial over a finite field, to which we will refer often.

Lemma 2.1. Let $S$ be a subset of $\mathrm{GF}(q)^{2}$ and $\sigma \in \mathrm{GF}(q)[X, Y]$ be such that $\sigma(a Y+b, Y) \equiv$ 0 , for all $(a, b) \in S$. If $|S|>\operatorname{deg}(\sigma)$ then $\sigma(X, Y) \equiv 0$.

Proof: If $\sigma(a Y+b, Y) \equiv 0$ then $\sigma(X, Y) \equiv 0$ modulo $X-a Y-b$, and hence

$$
X-a Y-b \mid \sigma(X, Y) \text {. }
$$

It follows that

$$
\prod_{(a, b) \in S}(X-a Y-b) \mid \sigma(X, Y)
$$

Since the degree of the left hand side is $|S|$ the result follows.

Theorem 2.2. Let $\mathcal{W} \subset \mathrm{AG}(3, q) \subset \mathrm{PG}(3, q), q=p^{h},|\mathcal{W}|=q^{2}$. If the number of directions not determined by $\mathcal{W}$ is at least $q$ then every plane of $\mathrm{PG}(3, q)$ meets $\mathcal{W}$ in 0 modulo p points.

Proof: Let $\pi$ denote the plane $X_{3}=0$ in $\mathrm{PG}(3, q), \mathcal{W}$ be contained in $\mathrm{PG}(3, q) \backslash \pi$, and $\mathcal{D}(\mathcal{W})$ denote the set of directions determined by $\mathcal{W}$. Choose a subset $\mathcal{U} \subset \pi \backslash \mathcal{D}(\mathcal{W})$ of size $q$. Without loss of generality we may assume that $\mathcal{U}=\left\{\left\langle 1, u_{i}, v_{i}, 0\right\rangle: i \in\{1, \ldots, q\}\right\}$. Consider the Rédei polynomial

$$
R(T, X, Y):=\prod_{\langle a, b, c, 1\rangle \in \mathcal{W}}(T+a X+b Y+c)=\sum_{j=0}^{q^{2}} \sigma_{j}(X, Y) T^{q^{2}-j} .
$$


Note that $\operatorname{deg}\left(\sigma_{j}\right) \leq j$. Since every line intersecting $\pi$ in a point of $\mathcal{U}$ contains at most one point of $\mathcal{W}$ and $|\mathcal{W}|=q^{2}$, every such line must intersect $\mathcal{W}$ in exactly one point. Consider

$$
\begin{aligned}
R\left(T,-u_{i} Y-v_{i}, Y\right) & =\prod_{\langle a, b, c, 1\rangle \in \mathcal{W}}\left(T+a\left(-u_{i} Y-v_{i}\right)+b Y+c\right) \\
& =\prod_{\langle a, b, c, 1\rangle \in \mathcal{W}}\left(T+\left(b-a u_{i}\right) Y+c-a v_{i}\right)
\end{aligned}
$$

The number of factors satisfying $b-a u_{i}=r$ and $c-a v_{i}=s$ is equal to the number of points of $\mathcal{W}$ on the line defined by the planes $X_{1}-u_{i} X_{0}=r X_{3}$ and $X_{2}-v_{i} X_{0}=s X_{3}$. Since this line is incident with the point $\left\langle 1, u_{i}, v_{i}, 0\right\rangle \in \mathcal{U}$, the number of such factors is one. Hence

$$
\begin{aligned}
R\left(T,-u_{i} Y-v_{i}, Y\right) & =\prod_{(r, s) \in \mathrm{GF}(q)^{2}}(T+r Y+s)=\prod_{r \in \mathrm{GF}(q)}\left(T^{q}+r Y^{q}-T-r Y\right) \\
& =T^{q^{2}}-\left(\left(Y^{q}-Y\right)^{q-1}+1\right) T^{q}+\left(Y^{q}-Y\right)^{q-1} T,
\end{aligned}
$$

for all $i \in\{1, \ldots, q\}$. It follows that $\sigma_{j}\left(-u_{i} Y-v_{i}, Y\right) \equiv 0$ for all $i \in\{1, \ldots, q\}, 0<j<$ $q^{2}-q$. By the previous lemma, $\sigma_{j}(X, Y) \equiv 0$ for $0<j<q$ since $\operatorname{deg}\left(\sigma_{j}\right) \leq j$. This implies that

$$
R(T, X, Y)=T^{q^{2}}+\sum_{j=q}^{q^{2}} \sigma_{j}(X, Y) T^{q^{2}-j}
$$

Differentiate the Rédei polynomial with respect to $T$

$$
\frac{\partial R}{\partial T}(T, X, Y)=\sum_{\langle a, b, c, 1\rangle \in \mathcal{W}} \frac{1}{(T+a X+b Y+c)} R(T, X, Y) .
$$

Evaluate at $X=x \in \mathrm{GF}(q)$ and $Y=y \in \mathrm{GF}(q)$ and multiply through by $T^{q}-T$. Then we have a polynomial identity and the divisibility

$$
R(T, x, y) \mid\left(T^{q}-T\right) \frac{\partial R}{\partial T}(T, x, y) .
$$

The left hand side has degree $q^{2}$ and the right hand side has degree less than $q^{2}$. Hence the right hand side is zero, in particular

$$
\frac{\partial R}{\partial T}(T, x, y) \equiv 0
$$

for all $(x, y) \in \operatorname{GF}(q)^{2}$. This implies that $R(T, x, y)$ is a $p$-th power, for all $(x, y) \in \operatorname{GF}(q)^{2}$. It follows that every factor $T-t$, where $t=-a x-b y-c$ for some $\langle a, b, c, 1\rangle \in \mathcal{W}$ occurs a multiple of $p$ times in $R(T, x, y)$. In other words, every plane with equation

$$
x X_{0}+y X_{1}+X_{2}+t X_{3}=0
$$


$x, y, t \in \mathrm{GF}(q)$, intersects $\mathcal{W}$ in 0 modulo $p$ points. These are all planes of $\mathrm{PG}(3, q)$ except those which have no $X_{2}$-term in their defining equation. But if we define the Rédei polynomial as

$$
\prod_{\langle a, b, c, 1\rangle \in \mathcal{W}}(T+a+b X+c Y)
$$

respectively

$$
\prod_{\langle a, b, c, 1\rangle \in \mathcal{W}}(T+a X+b+c Y)
$$

then exactly the same arguments as for $R(T, X, Y)$ can be applied and it follows that every plane of $\operatorname{PG}(3, q)$ intersects $\mathcal{W}$ in 0 modulo $p$ points, except those planes which have no $X_{0}$-term, respectively $X_{1}$-term, in their defining equation. The only plane belonging to all of the above exceptional planes is the plane $X_{3}=0$, which intersects $\mathcal{W}$ in 0 points.

The following example illustrates that the bound in Theorem 2.2 is sharp.

Example 2.3. Let $\pi$ and $\pi^{\prime}$ be two planes of $\mathrm{PG}(3, q), q=p^{h}$, intersecting in the line $L$. Suppose $P$ is a point of $\pi \backslash L, Q$ a point of $\pi^{\prime} \backslash L$ and $R$ a point on $L$. Define $\mathcal{W}$ as the set of points on $\pi^{\prime} \backslash L$ but not on the line $\langle Q, R\rangle$, together with the points on the line $\langle P, Q\rangle$ different from $P$. Then $\mathcal{W}$ has size $q^{2}, \mathcal{W}$ determines $q^{2}+2$ directions in $\pi$, the points on the line $\langle R, P\rangle \backslash\{R, P\}$ are not determined by $\mathcal{W}$, and not every plane intersects $\mathcal{W}$ in 0 modulo $p$ points.

In fact we can show that as the number of directions determined by $\mathcal{W}$ becomes smaller, the restriction on the intersection number with planes of $\mathrm{PG}(3, q)$ becomes stronger.

Theorem 2.4. Let $\mathcal{W} \subset \mathrm{AG}(3, q) \subset \mathrm{PG}(3, q), q=p^{h},|\mathcal{W}|=q^{2}$. If there are $p^{e} q$ or more directions not determined by $\mathcal{W}$ for some $e \in\{0,1,2, \ldots, h-1\}$ then every plane of $\mathrm{PG}(3, q)$ meets $\mathcal{W}$ in 0 modulo $p^{e+1}$ points.

Proof: The case $e=0$ was proven in Theorem 2.2 so assume that $e \geq 1$ and as in the proof of Theorem 2.2 let $\pi$ denote the plane $X_{3}=0$ in $\mathrm{PG}(3, q), \mathcal{W}$ be contained in $\mathrm{PG}(3, q) \backslash \pi$, and $\mathcal{D}(\mathcal{W})$ denote the set of directions determined by $\mathcal{W}$. Without loss of generality we may assume that $\langle 0,0,1,0\rangle \in \mathcal{D}(\mathcal{W})$ and by hypothesis there is a set $\mathcal{U} \subset \pi \backslash \mathcal{D}(\mathcal{W})$ of size $p^{e} q$. Put $\mathcal{U}=\left\{\left\langle 1, u_{i}, v_{i}, 0\right\rangle: i \in\left\{1, \ldots, p^{e} q-k\right\}\right\} \cup\left\{\left\langle 0,1, t_{i}, 0\right\rangle: i \in\{1, \ldots, k\}\right\}$. Consider the Rédei polynomial

$$
R(T, X, Y, Z):=\prod_{\langle a, b, c, 1\rangle \in \mathcal{W}}(T+a X+b Y+c Z)=\sum_{j=0}^{q^{2}} \sigma_{j}(X, Y, Z) T^{q^{2}-j} .
$$

Repeating the exact same arguments as in the proof of Theorem 2.2 but using the homogeneous polynomials $\sigma_{j}(X, Y, Z)$ we have that

$$
\sigma_{j}\left(-u_{i} Y-v_{i} Z, Y, Z\right) \equiv 0,
$$


for all $i$ and $0<j<q^{2}-q$. Hence, by an analogous result to Lemma 2.1

$$
\prod_{i=1}^{p^{e} q-k}\left(X+u_{i} Y+v_{i} Z\right) \mid \sigma_{j}(X, Y, Z)
$$

for $0<j<q^{2}-q$. Consider

$$
R\left(T, 1,-t_{i} Z, Z\right)=\prod_{\langle a, b, c, 1\rangle \in \mathcal{W}}\left(T+\left(c-t_{i} b\right) Z+a\right) .
$$

The number of factors satisfying $c-t_{i} b=r$ and $a=s$ is equal to the number of points of $\mathcal{W}$ on the line defined by the planes $X_{2}-t_{i} X_{1}=r X_{3}$ and $X_{0}=s X_{3}$. Since this line is incident with the point $\left\langle 0,1, t_{i}, 0\right\rangle$ the number of such factors is one. Hence

$$
\begin{aligned}
R\left(T, 1,-t_{i} Z, Z\right) & =\prod_{(r, s) \in \mathrm{GF}(q)^{2}}(T+r Z+s) \\
& =T^{q^{2}}-\left(\left(Z^{q}-Z\right)^{q-1}+1\right) T^{q}+\left(Z^{q}-Z\right)^{q-1} T,
\end{aligned}
$$

for all $i \in\{1, \ldots, k\}$. It follows that $\sigma_{j}\left(1,-t_{i} Z, Z\right) \equiv 0$ for all $i \in\{1, \ldots, k\}$ and $0<j<$ $q^{2}-q$. As in Lemma 2.1

$$
\prod_{i=1}^{k}\left(Y+t_{i} Z\right) \mid \sigma_{j}(X, Y, Z)
$$

and so

$$
\prod_{i=1}^{k}\left(Y+t_{i} Z\right) \prod_{i=1}^{p^{e} q-k}\left(X+u_{i} Y+v_{i} Z\right) \mid \sigma_{j}(X, Y, Z)
$$

for $0<j<q^{2}-q$. Now if $0<j<p^{e} q$ then the degree of $\sigma_{j}(X, Y, Z)$ is less than $p^{e} q$ and so $\sigma_{j}(X, Y, Z) \equiv 0$. Therefore

$$
R(T, X, Y, 1)=T^{q^{2}}+\sum_{j=p^{e} q}^{q^{2}} \sigma_{j}(X, Y, 1) T^{q^{2}-j} .
$$

and we can follow the proof of Theorem 2.2 and conclude that $R(T, x, y, 1)$ is a $p$-th power, for all $(x, y) \in \operatorname{GF}(q)^{2}$. Now fix an $(x, y) \in \operatorname{GF}(q)^{2}$ and take the $p$-th root of $R(T, x, y, 1)$, i.e.,

$$
R_{1}(T):=R(T, x, y, 1)^{1 / p}=T^{q^{2} / p}+G(T),
$$

for some $G \in \operatorname{GF}(q)[T]$, with $\operatorname{deg}(G) \leq\left(q^{2}-p^{e} q\right) / p$. Again, as in the proof of Theorem 2.2, we have that

$$
R_{1}(T) \mid\left(T^{q}-T\right) \frac{\partial R_{1}}{\partial T}(T)
$$


The left hand side has degree $q^{2} / p$ and the right hand side has degree at most $q^{2} / p+q-$ $p^{e} q / p-2<q^{2} / p$. Hence the right hand side is zero, in particular

$$
\frac{\partial R_{1}}{\partial T}(T) \equiv 0
$$

This implies that $R_{1}(T)$ is a $p$-th power and $R(T, x, y, 1)$ is a $p^{2}$-th power for all $(x, y) \in$ $\mathrm{GF}(q)^{2}$. We can continue this process by defining $R_{l}(T)$ as the $p^{l}$-th root of $R(T, x, y)$ for any fixed $(x, y) \in \operatorname{GF}(q)^{2}$, consider the divisibility

$$
R_{l}(T) \mid\left(T^{q}-T\right) \frac{\partial R_{l}}{\partial T}(T)
$$

and obtain that $R_{l}(T)$ is a $p$-th power, as long as the degree of the right hand side is less than $q^{2} / p^{l}$. This is the case as long as $l<e+1$, which implies that $R(T, x, y, 1)$ is a $p^{e+1}$-th power, for all $(x, y) \in \mathrm{GF}(q)^{2}$. It follows that every factor $T-t$, where $t=-a x-b y-c$ for some $\langle a, b, c, 1\rangle \in \mathcal{W}$, occurs a multiple of $p^{e+1}$ times in $R(T, x, y, 1)$. In other words, every plane with equation

$$
x X_{0}+y X_{1}+X_{2}+t X_{3}=0
$$

$x, y, t \in \mathrm{GF}(q)$, intersects $\mathcal{W}$ in 0 modulo $p^{e+1}$ points. These are all planes of $\operatorname{PG}(3, q)$ except those which have no $X_{2}$-term in their defining equation. However we can redefine the Rédei polynomial as in Theorem 2.2 , by permuting the coordinates, and conclude that all planes intersect $\mathcal{W}$ in 0 modulo $p^{e+1}$ points.

The following theorem says we can deduce more in the case when $q$ is even.

Theorem 2.5. Let $\mathcal{W} \subset \mathrm{AG}(3, q) \subset \mathrm{PG}(3, q), q=2^{h},|\mathcal{W}|=q^{2}$. Suppose that there are at least $2^{e} q$ directions not determined by $\mathcal{W}$ for some $e \in\{0,1, \ldots, h-1\}$. Then two parallel planes intersect $\mathcal{W}$ in the same number of points modulo $2^{e+2}$.

Proof: Put $\pi:=\mathrm{PG}(3, q) \backslash \mathrm{AG}(3, q)$ and suppose that $\pi_{1}$ and $\pi_{2}$ are two parallel planes intersecting $\pi$ in the same line determined by the equations $X_{3}=0$ and $x X_{0}+y X_{1}+X_{2}=0$ for some $x, y \in \operatorname{GF}(q)$. We assume that the planes $\pi_{1}$ and $\pi_{2}$ do not contain the point $\langle 0,0,1,0\rangle$, but as before we can permute the coordinates and consider planes that do not contain the point $\langle 1,0,0,0\rangle$ and the point $\langle 0,1,0,0\rangle$. Let

$$
\pi_{1}: x X_{0}+y X_{1}+X_{2}+t_{1} X_{3}=0
$$

and

$$
\pi_{2}: x X_{0}+y X_{1}+X_{2}+t_{2} X_{3}=0 \text {. }
$$

Theorem 2.4 implies that planes intersect $\mathcal{W}$ in zero modulo $2^{e+1}$ points.

Suppose $\pi_{1}$ intersects $\mathcal{W}$ in $2^{e+1} \bmod 2^{e+2}$ points. Then, as in the proof of Theorem 2.4, it follows that $t_{1}$ is a root of $R(T, x, y, 1)$, where $R(T, X, Y, Z)$ is the Rédei polynomial corresponding to $\mathcal{W}$, of multiplicity $2^{e+1} \bmod 2^{e+2}$, and we obtain $R(T, x, y, 1) \in$ $\mathrm{GF}(q)\left[T^{2^{e+1}}\right] \backslash \mathrm{GF}(q)\left[T^{2^{e+2}}\right]$. We will show that also $\pi_{2}$ intersects $\mathcal{W}$ in $2^{e+1} \bmod 2^{e+2}$ points. $\underline{\text { Springer }}$ 
We may write

$$
R(T, x, y, 1)^{1 / 2^{e+1}}=T^{q^{2} / 2^{e+1}}+g(T),
$$

where $g \in \mathrm{GF}(q)[T]$ is of degree at most $q^{2} / 2^{e+1}-q / 2$ and $g^{\prime}(T)$ is not identically zero. The product of the distinct linear factors of $R(T, x, y, 1)^{1 / 2^{e+1}}$ divides $T^{q}+T$ and the repeated factors divide its derivative, hence

$$
T^{q^{2} / 2^{e+1}}+g(T) \mid\left(T^{q}+T\right) g^{\prime}(T) .
$$

The degree of the quotient $m(T)$ is at most $q / 2-2$ and differentiating the identity

$$
\left(T^{q^{2} / 2^{e+1}}+g(T)\right) m(T)=\left(T^{q}+T\right) g^{\prime}(T),
$$

we get

$$
T^{q^{2} / 2^{e+1}} m^{\prime}(T)+(g(T) m(T))^{\prime}=g^{\prime}(T) .
$$

The degree of $g(T) m(T)$ is at most $q^{2} / 2^{e+1}-2$ so we must have that $m^{\prime}(T)=0$. The last equation then becomes $m(T) g^{\prime}(T)=g^{\prime}(T)$ and hence $m(T)=1$. Therefore

$$
R(T, x, y, 1)=\left(T^{q}+T\right)^{2^{e+1}} h(T)^{2^{e+2}},
$$

where $h(T)^{2}=g^{\prime}(T)$. It follows that every root of $R(T, x, y, 1)$, in particular $t_{2}$, is a root with multiplicity $2^{e+1} \bmod 2^{e+2}$, which implies that $\pi_{2}$ intersects $\mathcal{W}$ in $2^{e+1} \bmod 2^{e+2}$ points. We have shown that the number of points in the intersection of a plane with $\mathcal{W}$ modulo $2^{e+2}$ only depends on the plane's intersection with $\pi$.

\section{Ovoids of the generalised quadrangles $T_{2}(\mathcal{O})$ and $T_{2}^{*}(\mathcal{H})$}

Let $\mathcal{O}$ be an oval in $\mathrm{PG}(2, q) \subset \mathrm{PG}(3, q)$, i.e., a set of $q+1$ points no three collinear, where $q=p^{h}$. Consider the following incidence structure $T_{2}(\mathcal{O})$. We define three types of points: (i) the points of $\mathrm{PG}(3, q) \backslash \mathrm{PG}(2, q)$; (ii) The planes of $\mathrm{PG}(3, q)$ which meet $\mathrm{PG}(2, q)$ in a tangent line to $\mathcal{O}$; (iii) a point $(\infty)$. We define two type of lines: (a) the points of $\mathcal{O}$; (b) the lines of $\mathrm{PG}(3, q) \backslash \mathrm{PG}(2, q)$ which meet $\mathrm{PG}(2, q)$ in a point of $\mathcal{O}$. Incidence is symmetric containment in $\operatorname{PG}(3, q)$ and the point $(\infty)$ is incident with every line of type (a). The incidence structure $T_{2}(\mathcal{O})$ is a generalised quadrangle of order $q$, see [6,3.1.2]. An ovoid $\Omega$ of a generalised quadrangle $\mathcal{S}$ is a set of points of $\mathcal{S}$ such that every line of $\mathcal{S}$ is incident with exactly one point of $\Omega$. If the generalised quadrangle $\mathcal{S}$ has order $(s, t)$ then an ovoid of $\mathcal{S}$ has $s t+1$ points, again see [6]. Theorem 2.2 and Theorem 2.5 have the following immediate corollary.

Corollary 3.1. If $\Omega$ is an ovoid of $T_{2}(\mathcal{O})$ containing the point $(\infty)$, then every plane of $\mathrm{PG}(3, q)$ meets $\Omega$ in zero modulo $p$ points. Moreover if $q$ is even, two planes meeting $\mathrm{PG}(3, q) \backslash \mathrm{AG}(3, q)$ in the same line intersect $\Omega$ either both in 0 modulo 4 points or both in 2 modulo 4 points. 
Proof: Note that an ovoid of $T_{2}(\mathcal{O})$ contains $q^{2}+1$ points. The fact that no two points of $\mathcal{W}:=\Omega \backslash\{(\infty)\}$ are collinear means that the points of $\mathcal{O}$ are not contained in the set of directions determined by $\mathcal{W}$. Since $|\mathcal{W}|=q^{2}$ and $|\mathcal{O}|=q+1$, we can apply Theorem 2.2 and the first part of the corollary follows. The second part of the corollary follows directly from Theorem 2.5.

If $q$ is even then the oval $\mathcal{O}$ has a nucleus $N$, i.e., a point which is incident with every tangent line to $\mathcal{O}$. Consider the following incidence structure $T_{2}^{*}(\mathcal{H})$, where $\mathcal{H}=\mathcal{O} \cup\{N\}$. The points are the points of $\mathrm{PG}(3, q) \backslash \mathrm{PG}(2, q)$, the lines are the lines of $\mathrm{PG}(3, q) \backslash \mathrm{PG}(2, q)$ which meet $\mathrm{PG}(2, q)$ in a point of $\mathcal{H}$, and incidence is that inherited from $\operatorname{PG}(3, q) . T_{2}^{*}(\mathcal{H})$ is a generalised quadrangle of order $(q-1, q+1)$, see [6, 3.1.3]. Again we can apply Theorem 2.2 and Theorem 2.5 to obtain the following corollary for ovoids of $T_{2}^{*}(\mathcal{H})$.

Corollary 3.2. If $\Omega$ is an ovoid of $T_{2}^{*}(\mathcal{H})$, then every plane of $\mathrm{PG}(3, q)$ meets $\Omega$ in an even number of points. Moreover two planes meeting $\mathrm{PG}(3, q) \backslash \mathrm{AG}(3, q)$ in the same line intersect $\Omega$ either both in 0 modulo 4 points or both in 2 modulo 4 points.

Proof: Note that an ovoid of $T_{2}^{*}(\mathcal{H})$ has $(q-1)(q+1)+1=q^{2}$ points. The fact that no two points of $\mathcal{W}:=\Omega$ are collinear implies that the points of $\mathcal{H}$ are not contained in the set of directions determined by $\mathcal{W}$. Since $|\mathcal{W}|=q^{2}$ and $|\mathcal{H}|=q+2$, we can apply Theorem 2.2 and the first part of the corollary follows. The second part of the corollary follows directly from Theorem 2.5.

Motivated by the desire to know the possible intersection numbers that planes have with an ovoid of $T_{2}(\mathcal{O})$, where $(\infty)$ is not a point of the ovoid we prove the following theorem which would seem artificial were it not for the immediate corollary.

Theorem 3.3. Let $\mathcal{W} \subset \mathrm{AG}(3, q) \subset \mathrm{PG}(3, q), q=p^{h}$, be a set of $q^{2}-q$ point that does not determine a set of directions $\mathcal{U} \subset \pi \backslash \mathcal{D}(\mathcal{W})$, where $\pi:=\mathrm{PG}(3, q) \backslash \mathrm{AG}(3, q)$, which has the property that for each point $P \in \mathcal{U}$ the $q$ affine lines incident with $P$ but skew from $\mathcal{W}$ are coplanar.

(i) If $|\mathcal{U}| \geq q-1$ then two planes that meet $\pi$ in the same line are either both incident with a point of $\mathcal{W}$ or they are both incident with 0 modulo p points of $\mathcal{W}$.

(ii) If $\mathcal{U}$ is of size $q$ and has the property that the skew planes are incident with a common point $Q$ of $\pi$ then every plane not incident with $Q$ is incident with a point of $\mathcal{W}$ and those incident with $Q$ are incident with 0 modulo $p$ points of $\mathcal{W}$. Moreover if $q$ is even then every plane not incident with $Q$ is incident with an odd number of points of $\mathcal{W}$.

Proof: As before let $\pi$ denote the plane $X_{3}=0$ in $\mathrm{PG}(3, q), \mathcal{W}$ be contained in $\mathrm{PG}(3, q) \backslash$ $\pi$, and $\mathcal{D}(\mathcal{W})$ denote the set of directions determined by $\mathcal{W}$. Choose a subset $\mathcal{U} \subset \pi \backslash$ $\mathcal{D}(\mathcal{W})$ of size $q-1$. Without loss of generality we may assume that $\mathcal{U}=\left\{\left\langle 1, u_{i}, v_{i}, 0\right\rangle: i \in\right.$ $\{1, \ldots, q-1\}\}$. Define the Rédei polynomial

$$
R(T, X, Y):=\prod_{\langle a, b, c, 1\rangle \in \mathcal{W}}(T+a X+b Y+c)=\sum_{j=0}^{q^{2}-q} \sigma_{j}(X, Y) T^{q^{2}-q-j} .
$$




\section{Consider}

$$
R\left(T,-u_{i} Y-v_{i}, Y\right)=\prod_{\langle a, b, c, 1\rangle \in \mathcal{W}}\left(T+\left(b-a u_{i}\right) Y+c-a v_{i}\right)
$$

The number of factors satisfying $b-a u_{i}=r$ and $c-a v_{i}=s$ is equal to the number of points of $\mathcal{W}$ on the line defined by the planes $X_{1}-u_{i} X_{0}=r X_{3}$ and $X_{2}-v_{i} X_{0}=s X_{3}$. Since this line is incident with the point $\left\langle 1, u_{i}, v_{i}, 0\right\rangle \in \mathcal{U}$, the number of such factors is one unless the line is contained in the plane $\pi_{i}$ skew to $\mathcal{W}$ at $\left\langle 1, u_{i}, v_{i}, 0\right\rangle$. There is a point on the line $X_{3}=X_{0}=0$ that is not incident with any $\pi_{i}$ and without loss of generality we may assume that this point is $\langle 0,0,1,0\rangle$. So for some $\alpha_{i}, \beta_{i}$ the skew plane $\pi_{i}$ at $\left\langle 1, u_{i}, v_{i}, 0\right\rangle$ is defined by the equation

$$
-\left(v_{i}+\beta_{i} u_{i}\right) X_{0}+\beta_{i} X_{1}+X_{2}+\alpha_{i} X_{3}=0 .
$$

This plane contains the line defined by the equations $X_{1}-u_{i} X_{0}=r X_{3}$ and $X_{2}-v_{i} X_{0}=$ $s X_{3}$ if and only if $s=-\left(\alpha_{i}+\beta_{i} r\right)$. Hence

$$
\begin{aligned}
& R\left(T,-u_{i} Y-v_{i}, Y\right)=\prod_{(r, s) \in \mathrm{GF}(q)^{2}}(T+r Y+s) / \prod_{r \in \mathrm{GF}(q)}\left(T+r Y-\left(\alpha_{i}+\beta_{i} r\right)\right), \\
& \quad=\left[T^{q^{2}}-\left(\left(Y^{q}-Y\right)^{q-1}+1\right) T^{q}+\left(Y^{q}-Y\right)^{q-1} T\right] /\left[T^{q}-\left(Y-\beta_{i}\right)^{q-1} T-\alpha_{i}\right],
\end{aligned}
$$

for all $i \in\{1,2, \ldots, q-1\}$. The second highest degree term in $T$ on the right hand side is of degree $q^{2}-2 q+1$ so $\sigma_{j}\left(-u_{i} Y-v_{i}, Y\right) \equiv 0$ for all $j \in\{1,2, \ldots, q-2\}$ and $i \in$ $\{1,2, \ldots, q-1\}$. By Lemma 2.1 the polynomials $\sigma_{j}(X, Y) \equiv 0$ for all $j \in\{1,2, \ldots, q-2\}$. So

$$
R(T, X, Y)=T^{q^{2}-q}+\sum_{j=q-1}^{q^{2}-q} \sigma_{j}(X, Y) T^{q^{2}-q-j}
$$

As in the previous theorems for all $x, y \in \mathrm{GF}(q)$ we have the divisibility

$$
R(T, x, y) \mid\left(T^{q}-T\right) \frac{\partial R}{\partial T}(T, x, y) .
$$

The left hand side has degree $q^{2}-q$ and the right hand side has degree less than or equal to $q^{2}-q$. The coefficient of $T^{q^{2}-q}$ on the right hand side is $\sigma_{q-1}(x, y)$.

If $\sigma_{q-1}(x, y)$ is zero then the right hand side has degree less than the left hand side and is identically zero. In this case

$$
\frac{\partial R}{\partial T}(T, x, y) \equiv 0
$$

and $R(T, x, y)$ is a $p$-th power and it follows that every factor $T-t$, where $t=-a x-b y-c$ for some $\langle a, b, c, 1\rangle \in \mathcal{W}$ occurs a multiple of $p$ times in $R(T, x, y)$. In other words, every plane with equation

$$
x X_{0}+y X_{1}+X_{2}+t X_{3}=0
$$


$x, y, t \in \mathrm{GF}(q)$, intersects $\mathcal{W}$ in 0 modulo $p$ points. These are the planes sharing the common line of $\pi$ defined by the equations $X_{3}=0$ and $x X_{0}+y X_{1}+X_{2}=0$.

If $\sigma_{q-1}(x, y)$ is not zero then we have the equality

$$
R(T, x, y)=\sigma_{q-1}(x, y)^{-1}\left(T^{q}-T\right) \frac{\partial R}{\partial T}(T, x, y),
$$

and it follows that every factor $T-t$, where $t=-a x-b y-c$ for some $\langle a, b, c, 1\rangle \in \mathcal{W}$ occurs at least once in $R(T, x, y)$. In other words, every plane with equation

$$
x X_{0}+y X_{1}+X_{2}+t X_{3}=0
$$

$x, y, t \in \mathrm{GF}(q)$, intersects $\mathcal{W}$ in at least a point. Again these planes share the common line of $\pi$ defined by the equations $X_{3}=0$ and $x X_{0}+y X_{1}+X_{2}=0$ and so we have proved the first part of the theorem for all lines which have an $X_{2}$ term in their defining equation. As in the previous theorems, redefining the Rédei polynomial by permuting the coordinates and going through the same arguments suffices for lines of $\pi$ defined by equations of the form $x X_{0}+X_{1}+y X_{2}=0$ and $X_{0}+x X_{1}+y X_{2}=0$.

By hypothesis in the final part of the theorem we have a subset of $\mathcal{U} \subset \pi \backslash \mathcal{D}(\mathcal{W})$ of size $q$ with the property that the planes skew to $\mathcal{W}$ are incident with a common point $Q$ of $\pi$. Then every plane not incident with $Q$ is incident with a point of $\mathcal{W}$. Without loss of generality let $Q$ be the point $\langle 0,1,0,0\rangle$ and apply a collineation that fixes $Q$ and maps the line $X_{0}=0$ skew to $\mathcal{U}$. Following the proof as in part (i), but with $\beta_{i}=0$ for all $i \in\{1,2, \ldots, q\}$ we have

$$
\begin{aligned}
R\left(T,-u_{i} Y-v_{i}, Y\right)= & \left(T^{q^{2}}-\left(\left(Y^{q}-Y\right)^{q-1}+1\right) T^{q}\right. \\
& \left.+\left(Y^{q}-Y\right)^{q-1} T\right) /\left(T^{q}-Y^{q-1} T-\alpha_{i}\right),
\end{aligned}
$$

for all $i \in\{1,2, \ldots, q\}$. Hence $\sigma_{q-1}\left(-u_{i} Y-v_{i}, Y\right) \equiv Y^{q-1}$ and by Lemma 2.1 $\sigma_{q-1}(X, Y)-Y^{q-1} \equiv 0$. Continuing along the arguments as before we now have that if $y \neq 0$ then the every plane with equation

$$
x X_{0}+y X_{1}+X_{2}+t X_{3}=0
$$

$x, t \in \mathrm{GF}(q)$, intersects $\mathcal{W}$ in at least a point and if $y=0$ then the planes defined by an equation of the form

$$
x X_{0}+X_{2}+t X_{3}=0
$$

those incident with $Q$, intersect $\mathcal{W}$ in 0 modulo $p$ points. Moreover, if $q$ is even and $y \neq 0$ then

$$
R(T, x, y)=\sigma_{q-1}(x, y)^{-1}\left(T^{q}-T\right) \frac{\partial R}{\partial T}(T, x, y)
$$

Since $\frac{\partial R}{\partial T}(T, x, y)$ is a square in $T$ every factor $T-t$ occurs an odd number of times and the planes defined by an equation of the form

$$
x X_{0}+y X_{1}+X_{2}+t X_{3}=0
$$

intersect $\mathcal{W}$ in an odd number of points. 
Corollary 3.4. Let $\Omega$ be an ovoid of $T_{2}(\mathcal{O})$ that does not contain the point $(\infty)$. Every plane of $P G(3, q)$ that is not incident with a point of $\mathcal{O}$ is incident with 1 modulo $p$ points of $\Omega$.

Proof: If $q$ is even then all the hypotheses of Theorem 3.3 are satisfied and we can apply the last part of the theorem to obtain the corollary. If $q$ is odd then $\mathcal{O}$ is a conic and $T_{2}(\mathcal{O})$ is isomorphic to $Q(4, q)$. The planes of $P G(3, q)$ that are not incident with a point of $\mathcal{O}$ correspond to elliptic quadrics in the $Q(4, q)$ model. Corollary 3.1 implies that elliptic quadrics are incident with no points of an ovoid of $Q(4, q)$ or 1 modulo $p$ points. However Theorem 3.3 shows that the planes containing the line $\pi^{\prime} \cap \pi$, where $\pi^{\prime}$ is a plane skew to the ovoid, are all skew to the ovoid, which is clearly nonsense. Hence an elliptic quadric is incident with 1 modulo $p$ points of an ovoid of $Q(4, q)$.

In the case when $q$ is odd, the previous corollary was first proven in [3]. It was proven again in [4] where it was also shown that ovoids of $Q(4, p), p$ prime, are elliptic quadrics.

In the case where $q$ is even and $\mathcal{O}$ is a conic, so $T_{2}(\mathcal{O})$ is isomorphic to $Q(4, q)$, the previous corollary was first proven by Bagchi and Sastry [1]. Moreover it was shown in [4] that every elliptic quadric is either incident with 1 modulo 4 points of an ovoid of $Q(4, q)$ or every elliptic quadric is incident with 3 modulo 4 points of an ovoid of $Q(4, q)$.

Acknowledgment S. Ball acknowledges the support of the Ministerio de Ciencia y Tecnologia, España. M. Lavrauw acknowledges the financial support provided through the European Community's Human Potential Programme under contract HPRN-CT-2002-00278, COMBSTRU.

\section{References}

1. B. Bagchi and N. S. Narasimha Sastry, "Even order inversive planes, generalized quadrangles and codes," Geom. Dedicata, 22 (1987) 137-147.

2. S. Ball, "The number of directions determined by a function over a finite field," J. Combin. Theory Ser. A, 104 (2003) 341-350.

3. S. Ball, "On ovoids of $O(5, q), " A d v$. Geom., 4 (2004) 1-7.

4. S. Ball, P. Govaerts, and L. Storme, "On ovoids of parabolic quadrics," Des. Codes Cryptogr., 38 (2006) $131-145$.

5. A. Blokhuis, S. Ball, A.E. Brouwer, L. Storme, and T. Szőnyi, "On the number of slopes of the graph of a function defined over a finite field," J. Combin. Theory Ser. A, 86 (1999) 187-196.

6. S.E. Payne, and J.A. Thas, Finite Generalized Quadrangles. Research Notes in Mathematics, 110. Pitman (Advanced Publishing Program), Boston, MA, 1984. vi+312 pp. ISBN 0-273-08655-3

7. L. Rédei, Lacunary Polynomials Over Finite Fields, North-Holland, Amsterdam, 1973.

8. L. Storme and P. Sziklai, "Linear point sets and Rédei type $k$-blocking sets in $P G(n, q)$," J. Algebraic Combin., 14 (2001) 221-228. 